\title{
Expression of breast cancer stem cell markers as predictors of prognosis and response to trastuzumab in HER2-positive breast cancer
}

\author{
An Na Seo ${ }^{1,2,11}$, Hee Jin Lee ${ }^{1,3,11}$, Eun Joo Kim¹, Min Hye Jang ${ }^{1}$, Yu Jung Kim ${ }^{4,5}$, Jee Hyun Kim ${ }^{4,5}$, \\ Sung-Won Kim ${ }^{6,7}$, Han Suk Ryu ${ }^{8}$, In Ae Park ${ }^{8}$, Seock-Ah Im ${ }^{5}$, Gyungyub Gong ${ }^{3}$, Kyung Hae Jung ${ }^{9}$, \\ Hee Jeong $\mathrm{Kim}^{10}$ and So Yeon Park ${ }^{*}, 1,8$
}

\begin{abstract}
${ }^{1}$ Department of Pathology, Seoul National University Bundang Hospital, 300 Gumi-dong, Bundang-gu, Seongnam-si, Gyeonggi 463-707, Korea; ${ }^{2}$ Department of Pathology, Kyungpook National University Hospital, Kyungpook National University School of Medicine, 680 Gukchaebosang-ro, Jung-gu, Daegu 700-842, Korea; ${ }^{3}$ Department of Pathology, Asan Medical Center, 88 Olympic-ro 43-gil, Songpa-gu, Seoul 138-746, Korea; ${ }^{4}$ Department of Medical Oncology, Seoul National University Bundang Hospital, 300 Gumi-dong, Bundang-gu, Seongnam-si, Gyeonggi 463-707, Korea; ${ }^{5}$ Department of Medical Oncology, Seoul National University College of Medicine, 28 Yeongon-dong, Jongno-gu, Seoul 110-799, Korea; ${ }^{6}$ Department of Surgery, Seoul National University Bundang Hospital, 300 Gumi-dong, Bundang-gu, Seongnam-si, Gyeonggi 463-707, Korea; ${ }^{7}$ Department of Surgery, Seoul National University College of Medicine, 28 Yeongon-dong, Jongno-gu, Seoul 110-799, Korea; ${ }^{8}$ Department of Pathology, Seoul National University College of Medicine, 28 Yeongon-dong, Jongno-gu, Seoul 110-799, Korea; ${ }^{9}$ Department of Medical Oncology, Asan Medical Center, 88 Olympic-ro 43-gil, Songpa-gu, Seoul 138-746, Korea and ${ }^{10}$ Department of Surgery, Asan Medical Center, 88 Olympic-ro 43-gil, Songpa-gu, Seoul 138-746, Korea
\end{abstract}

Background: Breast cancer stem cells (BCSCs) have been suggested to have clinical implications for cancer therapeutics because of their proposed role in chemoresistance. The aim of this study was to investigate the impact of BCSC marker expression on clinical outcome and trastuzumab response in human epidermal growth factor receptor 2 (HER2)-positive breast cancer.

Methods: We analysed the expression of BCSC markers, CD44 + /CD24 - and aldehyde dehydrogenase 1 (ALDH1), and clinical outcomes in three sets of breast cancer cases: Set 1, 242 HER2-positive primary breast cancers treated by various modalities; Set 2, 447 HER2-positive primary breast cancers treated with surgery and chemotherapy plus adjuvant trastuzumab; Set 3, 112 metastatic HER2-positive breast cancers treated with trastuzumab.

Results: Expression of CD44 +/CD24 - and ALDH1 was detected in $30.7 \%$ and 10.0\%, respectively, of the Set 1 cases, and was associated with hormone receptor negativity. In survival analyses, expression of CD44 + /CD24 - , but not ALDH1, was found to be an independent prognostic factor for poor disease-free and overall survival in whole patients and also in the subgroup not receiving adjuvant trastuzumab. In Set 2 cases treated with adjuvant trastuzumab, CD44 +/CD24- expression was an independent prognostic factor for poor disease-free survival, but not for overall survival; expression of ALDH1 had no impact on disease-free or overall survival. In metastatic disease treated with trastuzumab (Set 3 cases), CD44+/CD24- and ALDH1 expression had no effect on trastuzumab response or survival.

Conclusions: These results suggest that the CD44 +/CD24 - phenotype can be used as a prognostic factor for clinical outcome and a predictive factor of trastuzumab response in patients with HER2-positive primary breast cancer.

\footnotetext{
*Correspondence: Dr SY Park; E-mail: sypmd@snu.ac.kr

${ }^{11}$ These authors contributed equally to this work.
}

Received 2 March 2016; revised 11 March 2016; accepted 22 March 2016; published online 26 April 2016

(c) 2016 Cancer Research UK. All rights reserved 0007-0920/16 
Human epidermal growth factor receptor 2 (HER2) plays a key role in the carcinogenesis of HER2-positive breast cancers that comprise $15-20 \%$ of human breast cancers and are associated with aggressive clinical behaviour and poor clinical outcome (Slamon et al, 1987; Tandon et al, 1989; Press et al, 1993). Development of agents such as trastuzumab that target HER2 in treatment of HER2-positive breast cancer is one of the greatest achievements in clinical oncology. Consequently, there has been a significant decrease in the mortality because of this disease during the past 20 years (Slamon et al, 2001; Romond et al, 2005). However, despite the clinical efficacy of HER2-targeted therapy, many patients still experience recurrence. Unfortunately, patients who receive trastuzumab do not respond uniformly to the therapeutics, and $\sim 15 \%$ of patients with HER2-positive breast cancer show de novo resistance to trastuzumab and relapse in spite of treatment (Bedard et al, 2009). Many attempts have been made to find the predictors of response to HER2-targeted therapy. Several possible mechanisms of resistance to trastuzumab have been proposed, such as impaired access of trastuzumab to the receptor HER2, alternative signalling from the insulin-like growth factor-1 receptor or other epidermal growth factor receptors and aberrant activation of downstream signalling via PIK3CA mutation, deletion of phosphatase and tensin homologue (PTEN) on chromosome 10, and downregulation of p27 (Nahta et al, 2006; Mukohara, 2011).

In addition to intertumoral heterogeneity in treatment response, there is significant intertumoral cellular heterogeneity within the population of breast cancer cells in individual patients (Bedard et al, 2009). Accumulating evidence suggests that a subset of tumour cells that exhibit stem cell properties, the so-called cancer stem cells (CSCs), have the capacity to self-renew and differentiate, resulting in generation of a heterogeneous population of cells (Clarke and Fuller, 2006; Polyak and Hahn, 2006). It has been suggested that CSCs mediate metastatic dissemination, and contribute to chemoresistance, resulting in tumour recurrence and relapse (Clarke and Fuller, 2006; Kakarala and Wicha, 2008; Polyak and Hahn, 2006). Breast CSCs (BCSCs) can be identified through protein expression of their surrogate markers, such as CD44 + /CD24 - and aldehyde dehydrogenase 1 (ALDH1) (Al-Hajj et al, 2003; Ginestier et al, 2007). In breast cancer, it has been demonstrated that BCSCs are associated with basal-like breast cancer and adverse clinical outcome (Nalwoga et al, 2010; Park et al, 2010; Zhou et al, 2010). Moreover, some studies have found an association between BCSCs and chemoresistance ( $\mathrm{Li}$ et al, 2008; Tanei et al, 2009; Lee et al, 2011).

Recent observations suggest that HER2-targeted therapy, such as with trastuzumab, may directly target BCSCs in HER2-positive breast cancers (Bedard et al, 2009; Martin-Castillo et al, 2013). In contrast, it was also suggested that clinical resistance to trastuzumab may be driven by BCSCs (Bedard et al, 2009; Martin-Castillo et al, 2013). Korkaya et al (2008) showed that trastuzumab decreased the ALDEFLUOR-positive BCSC population in trastuzumab-sensitive, HER2-amplified breast cancer cell lines through inhibition of PI3K/Akt signalling, but not in trastuzumab-resistant cell lines. Furthermore, dynamic emergence of biologically aggressive CD44+/CD24mesenchymal progenies was found in a trastuzumab-refractory JIMT-1 breast cancer cell line, suggesting de novo resistance to trastuzumab in basal-like/HER2-positive breast cancer (Oliveras-Ferraros et al, 2010). However, the effect of BCSC marker expression on trastuzumab response has not been fully elucidated in HER2-positive human breast cancer tissue samples.

In this study, we examined the immunohistochemical expression of CD44 + /CD24 - and ALDH1 as surrogate markers for BCSCs in HER2-positive primary breast cancer to investigate their clinicopathologic significance and prognostic value. We also evaluated the correlation of BCSC marker expression with trastuzumab response and clinical outcome in HER2-positive primary and metastatic breast cancer treated with trastuzumab.

\section{MATERIALS AND METHODS}

Patients and samples. This retrospective study was conducted in Seoul National University Bundang Hospital in collaboration with the Asan Medical Center and Seoul National University Hospital. Three independent sets of cases described in a previous study (Lee et al, 2014b) were used. Briefly, Set 1 comprised 242 cases of HER2-positive primary breast cancer that were composed of 105 cases operated on at Seoul National University Bundang Hospital between 2003 and 2009, and 137 cases operated on at Asan Medical Center (AMC) between 2003 and 2004. Set 2 comprised 447 cases of HER2-positive primary breast cancer treated with surgery, chemotherapy, and 1 year of adjuvant trastuzumab at AMC between 2006 and 2011. Set 3 comprised 112 cases of HER2-positive metastatic breast cancer treated with trastuzumab at Seoul National University Bundang Hospital, Seoul National University Hospital, and AMC between 2001 and 2011. Of the 112 HER2-positive metastatic breast cancers, 3 from Seoul National University Bundang Hospital were also included in Set 1 as primary tumours. Further information on each set of cases is provided in detail in a previous study (Lee et al, 2014b). The present study was approved by the institutional review boards of all participating institutions and was exempted from the need for informed consent.

Immunohistochemical staining and scoring of BCSCs. Tissue microarrays of the three sets of cases and whole sections of biopsy specimens from the cases with metastasis were stained in a BenchMark XT autostainer (Ventana Medical Systems, Tucson, AZ, USA). Briefly, tissue sections $4 \mu \mathrm{m}$ thick were cut, dried, deparaffinised, and rehydrated following standard procedures. All sections were subjected to a heat-induced antigen retrieval process. After optimisation using positive and negative controls for each marker, preparations were stained with antibodies against CD44 (156-3C11 diluted 1:1000; Thermo Scientific, Fremont, CA, USA), CD24 (SN3b diluted 1:100; Thermo Scientific), ALDH1 (44 diluted 1:100; BD Biosciences, San Jose, CA, USA), and cytokeratin 5/6 (CK5/6) (D5/16 B4 diluted 1:50; Dako, Carpinteria, CA, USA) using an UltraView detection kit (Ventana Medical Systems).

Double immunostaining with the EnVision G|2 Doublestain System, Rabbit/Mouse (DAB + /Permanent Red) (Dako) was used to detect CD44 +/CD24 - cells according to the manufacturer's protocols. CD44 was detected with diaminobenzidine and CD24 with Permanent Red. Expression of CD $44+/ \mathrm{CD} 24-$ and ALDH1 was scored as the percentage of positive tumour cells, in $10 \%$ increments, and cases with $10 \%$ or greater staining were considered positive.

For cytokeratin 5/6, cases with any positive membranous staining were grouped as positive. Expression data of EGFR were extracted from a previous study (Lee et al, 2014b), and expression of either CK5/6 or EGFR ( $1+$ or more) was defined as basal-like phenotype.

Statistical analyses and assessment of trastuzumab response. The software Statistical Package for the Social Sciences version 18.0 (SPSS Inc., Chicago, IL, USA) was used for all analyses. Association between expression of BCSC markers and clinicopathologic variables was assessed using $\chi^{2}$ or Fisher's exact tests for categorical variables and $t$-test for continuous variables. The correlation between ALDH1 expression and CD44 + /CD24 - phenotype was determined using Spearman's correlation analysis.

Survival curves were estimated using the Kaplan-Meier method, and the significance of differences was assessed using the log-rank 
test. In Set 1 and Set 2, disease-free survival was calculated from the date of surgery to the date of locoregional recurrence, distant metastasis, or death caused by breast cancer as the first event. Overall survival was estimated from the date of surgery to the date of death from any cause. In the cases with metastatic disease, responses to trastuzumab-based therapy were evaluated every 8 to 12 weeks using the Response Evaluation Criteria in Solid Tumours (RECIST) version 1.1 (Eisenhauer et al, 2009), as previously reported (Lee et al, 2014b, c). Clinical benefit was defined as complete response, partial response, or stable disease for at least 6 months. Time to progression was defined as the time from initiation of trastuzumab treatment to disease progression, and overall survival as the time from initiation of trastuzumab treatment to death from any cause.

For multivariate analysis, the Cox proportional hazards regression model was used with a backward stepwise selection method, with covariates that were significantly associated with patient outcome in the univariate analyses. The hazard ratio (HR) and its 95\% confidence interval (CI) were calculated for each factor. Statistical significance was set at $P<0.05$ for each analysis and all $P$-values reported were two sided.

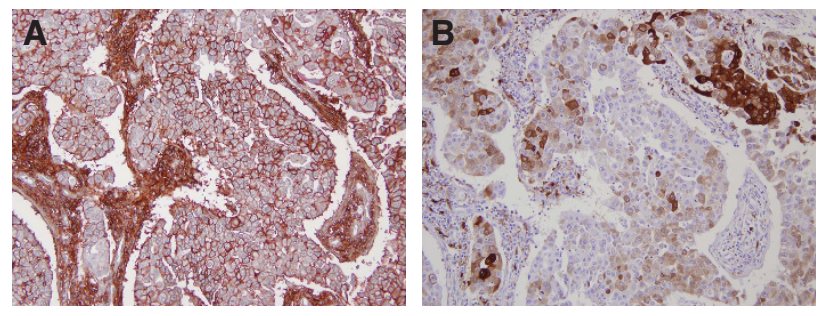

Figure 1. CD44 + /CD24 - and ALDH1 expression in HER2-positive breast cancer. A representative case showing CD44 + /CD24 - (A) (CD44: brown, CD24: red) and ALDH1 (B) expression.

\section{RESULTS}

Clinicopathologic features of HER2-positive primary breast cancer with BCSC marker expression in Set 1. In the cases in Set 1 , the BCSC markers, CD $44+/ C D 24-$ and ALDH1, were assessed immunohistochemically in 241 of 242 HER2-positive primary breast cancers (the tissue microarray core was lost in one case). Of these cases, $74(30.7 \%)$ had the CD $44+/ \mathrm{CD} 24-$ phenotype and $24(10.0 \%)$ were ALDH1 positive (Figure 1). Expressions of these two markers were positively correlated (rho $=0.229, P<0.001)$. The clinicopathologic characteristics of Set 1 in relation to BCSC marker expression are shown in Table 1. Expression of $\mathrm{CD} 44+/ \mathrm{CD} 24-$ was associated with hormone receptor negativity $(P=0.005)$ and basal-like phenotype $(P=0.003)$; ALDH1 positivity was associated with hormone receptor negativity $(P=0.002)$ and tended to be associated with low $\mathrm{T}$ stage $(P=0.073)$ and basal-like phenotype $(P=0.056)$. However, expression of CD44+/CD24- and ALDH1 did not show any association with $\mathrm{pN}$ stage, histologic grade, lymphovascular invasion, p53 overexpression, and $\mathrm{Ki}-67$ proliferation index.

Survival analysis according to BCSC marker expression in Set 1. In Set 1, the median follow-up period was 118.1 (0.7-171.5) months, during which there were $46(19.0 \%)$ recurrences and 48 (19.8\%) deaths. In survival analyses, CD $44+/ C D 24-$ expression was significantly associated with worse disease-free and overall survival ( $P=0.004$ and $P=0.014$, respectively; Figure 2). Although ALDH1 positivity tended to be associated with decreased disease-free and overall survival of the patients, the effect did not reach statistical significance $(P=0.125$ and $P=0.159$, respectively; Figure 2). In multivariate analyses including $\mathrm{CD} 44+/ \mathrm{CD} 24-$ expression, $\mathrm{pT}$ stage, $\mathrm{pN}$ stage, and lymphovascular invasion, which

Table 1. Clinicopathologic characteristics of HER2-positive primary breast cancers according to BCSC marker expression in Set 1

\begin{tabular}{|c|c|c|c|c|c|c|}
\hline \begin{tabular}{|l} 
Clinicopathologic characteristics \\
\end{tabular} & \multicolumn{2}{|c|}{ CD44+/CD24 - expression } & \multicolumn{4}{|c|}{ ALDH1 expression } \\
\hline \multicolumn{7}{|l|}{ pT stage } \\
\hline $\mathrm{T} 2-\mathrm{T} 4$ & $91(54.5)$ & $41(55.4)$ & 0.895 & $123(56.7)$ & $9(37.5)$ & 0.073 \\
\hline \multicolumn{7}{|l|}{ pN stage } \\
\hline NO & $91(54.5)$ & $35(47.3)$ & 0.302 & $115(53.0)$ & $11(45.8)$ & 0.505 \\
\hline I and II & $52(31.1)$ & $18(24.3)$ & \multirow[t]{2}{*}{0.282} & $65(30.0)$ & $5(20.8)$ & \multirow[t]{2}{*}{0.350} \\
\hline III & $115(68.9)$ & $56(75.7)$ & & $152(70.0)$ & $19(79.2)$ & \\
\hline \multicolumn{7}{|l|}{ Lymphovascular invasion } \\
\hline Negative & 104 (52.3) & 41 (55.4) & \multirow[t]{2}{*}{0.315} & $131(60.4)$ & 14 (58.3) & \multirow[t]{2}{*}{0.847} \\
\hline Positive & $63(3.7)$ & $33(44.6)$ & & $86(39.6)$ & $10(41.7)$ & \\
\hline Negative & 90 (53.9) & 41 (55.4) & \multirow[t]{2}{*}{0.828} & $117(53.9)$ & $14(58.3)$ & \multirow[t]{2}{*}{0.680} \\
\hline Positive & $77(46.1)$ & $33(44.6)$ & & $100(46.1)$ & $10(41.7)$ & \\
\hline \multicolumn{7}{|l|}{ Ki67 index } \\
\hline$<20 \%$ & $51(30.5)$ & $18(24.3)$ & \multirow[t]{2}{*}{0.325} & $62(28.6)$ & $7(29.2)$ & \multirow[t]{2}{*}{0.951} \\
\hline$\geqslant 20 \%$ & $116(69.5)$ & $56(75.7)$ & & $155(71.4)$ & $17(70.8)$ & \\
\hline \multicolumn{7}{|l|}{ Basal-like phenotype } \\
\hline Negative & $109(66.1)$ & $34(45.9)$ & \multirow[t]{2}{*}{0.003} & $133(61.9)$ & $10(41.7)$ & \multirow[t]{2}{*}{0.056} \\
\hline Positive & $56(33.9)$ & $40(54.1)$ & & $82(38.1)$ & $14(58.3)$ & \\
\hline
\end{tabular}


A

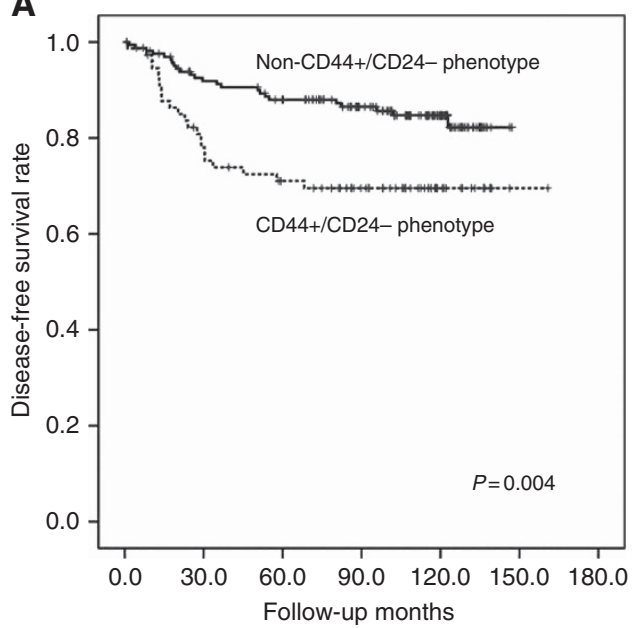

C

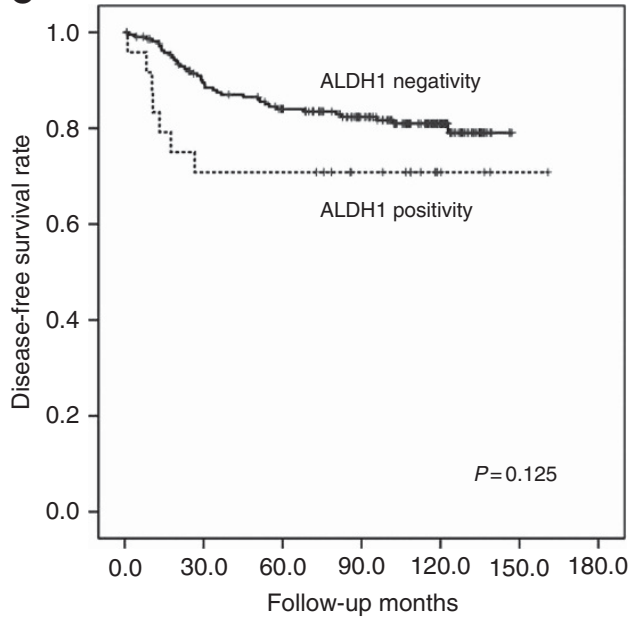

B

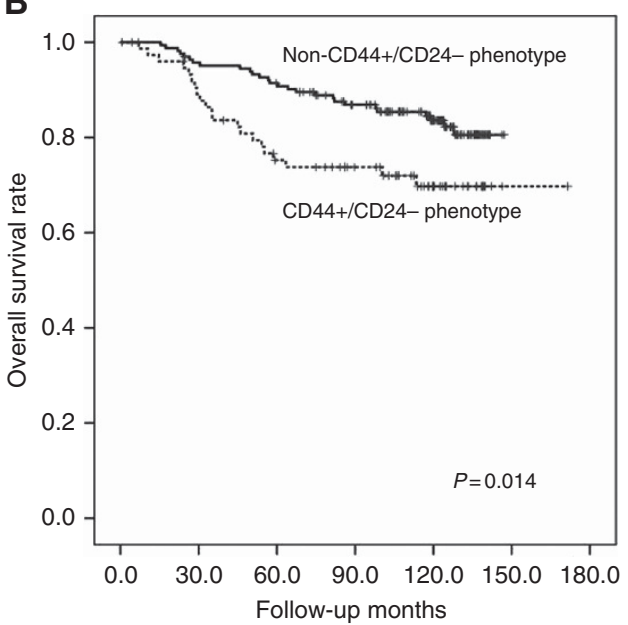

D

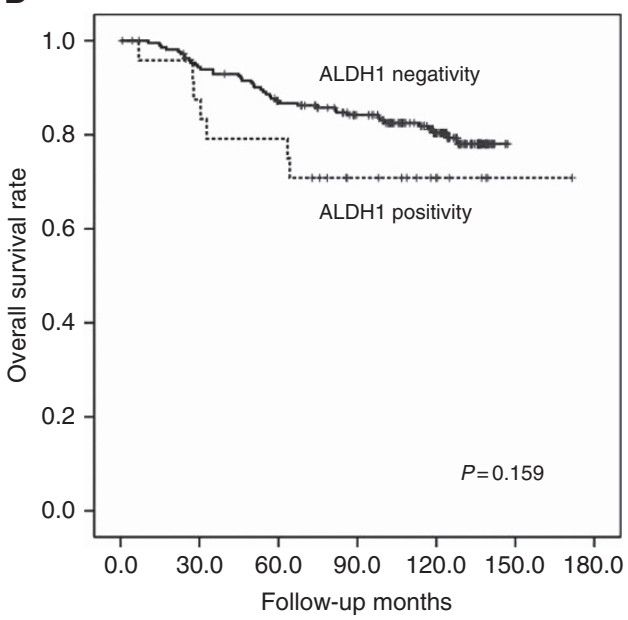

Figure 2. Kaplan-Meier plots of survival according to expression of CD44 +/CD24 - and ALDH1 in HER2-positive primary breast cancer treated by various modalities (Set 1). The CD44 +/CD24 - phenotype is significantly associated with poor disease-free and overall survival of the patients (A and $\mathbf{B})$. The ALDH1 positivity tends to be associated with decreased disease-free and overall survival of the patients (C and $\mathbf{D})$.

Table 2. Univariate and multivariate analyses of survival in HER2-positive primary breast cancer patients in Set 1

\begin{tabular}{|c|c|c|c|c|c|c|c|}
\hline \multirow[b]{2}{*}{ Variable } & \multirow[b]{2}{*}{ Category } & \multicolumn{3}{|c|}{ Univariate analysis } & \multicolumn{3}{|c|}{ Multivariate analysis } \\
\hline & & HR & $95 \% \mathrm{Cl}$ & $P$-value & HR & $95 \% \mathrm{Cl}$ & $P$-value \\
\hline \multicolumn{8}{|l|}{ Disease-free survival } \\
\hline pT stage & T1 vs T2-4 & 2.018 & $1.076-3.783$ & 0.025 & 1.582 & $0.834-3.003$ & 0.160 \\
\hline pN stage & N0 vs N1-3 & 3.947 & $2.004-7.774$ & $<0.001$ & 3.817 & $1.936-7.524$ & $<0.001$ \\
\hline Lymphovascular invasion & Absent vs present & 1.764 & $0.989-3.146$ & 0.051 & 0.879 & $0.460-1.680$ & 0.696 \\
\hline Hormone receptor status & Negative vs positive & 0.741 & $0.400-1.374$ & 0.340 & 0.882 & $0.436-1.784$ & 0.727 \\
\hline Basal-like phenotype & Negative vs positive & 1.714 & $0.961-3.058$ & 0.065 & 1.247 & $0.685-2.270$ & 0.470 \\
\hline CD44 + /CD24 - expression & Negative vs positive & 2.290 & $1.283-4.085$ & 0.004 & 2.143 & $1.201-3.826$ & 0.010 \\
\hline \multicolumn{8}{|l|}{ Overall survival } \\
\hline pT stage & T1 vs T2-4 & 1.528 & $0.846-2.761$ & 0.157 & 1.190 & $0.650-2.178$ & 0.572 \\
\hline pN stage & No vs N1-3 & 3.644 & $1.896-7.006$ & $<0.001$ & 3.525 & $1.832-6.786$ & $<0.001$ \\
\hline Lymphovascular invasion & Absent vs present & 1.762 & $1.000-3.105$ & 0.047 & 0.980 & $0.520-1.846$ & 0.949 \\
\hline Hormone receptor status & Positive vs negative & 0.813 & $0.446-1.481$ & 0.498 & 0.856 & $0.466-1.570$ & 0.614 \\
\hline Basal-like phenotype & Negative vs positive & 1.207 & $0.682-2.135$ & 0.517 & 1.174 & $0.615-2.245$ & 0.627 \\
\hline CD44 + /CD24 - expression & Negative vs positive & 2.018 & $1.140-3.572$ & 0.014 & 1.864 & $1.052-3.303$ & 0.033 \\
\hline
\end{tabular}

were associated with clinical outcome in the univariate analyses, and hormone receptor status and basal-like phenotype, which were closely associated with CD $44+/ \mathrm{CD} 24-$ expression, CD $44+1$ CD24 - expression was found to be an independent predictor of poor disease-free survival $(\mathrm{HR}=2.143,95 \% \mathrm{CI}=1.201-3.826$,
$P=0.010)$ and overall survival $(\mathrm{HR}=1.864,95 \% \mathrm{CI}=1.052-$ 3.303, $P=0.033$ ) along with lymph node metastasis (Table 2).

The patients in Set 1 were not evenly treated, and thus we performed subgroup analyses according to adjuvant trastuzumab therapy. In the subgroup not receiving adjuvant trastuzumab 
$(n=208)$, CD $44+/ C D 24-$ phenotype also showed a significant association with poor disease-free survival and overall survival ( $P=0.008$ and $P=0.029$, respectively). The ALDH1 positivity tended to be associated with decreased disease-free and overall survival of the patients $(P=0.128$ and $P=0.152$, respectively). In multivariate analyses, CD $44+/ \mathrm{CD} 24-$ phenotype $(\mathrm{HR}=2.135$, 95\% CI $=1.164-3.914, P=0.014)$ and lymph node metastasis $(\mathrm{HR}=4.203,95 \% \mathrm{CI}=2.063-8.561, P<0.001)$ were revealed as independent prognostic factors for poor disease-free survival, but only lymph node metastasis $(\mathrm{HR}=3.842,95 \% \mathrm{CI}=1.939-7.613$, $P<0.001)$ was an independent prognostic factor for overall survival (Supplementary Table S1).

However, because of the small sample size $(n=33)$, we could not determine the prognostic and predictive significance of BCSC marker expression in the subgroup treated with adjuvant trastuzumab.

Association of BCSC marker expression with clinical outcome in Set 2 cases. To further investigate the utility of BCSC marker expression as a predictive marker for trastuzumab response in HER2-positive breast cancer, we also analysed expression of CD44+/CD24 - and ALDH1 in an independent set of 447 patients with HER2-positive primary breast cancer treated with adjuvant trastuzumab (Set 2). Immunohistochemical assessment was possible in 435 cases (tissue microarray cores were lost in 12 cases). Of the 435 cases, 175 (40.2\%) showed CD $44+/ C D 24-$ phenotype and $40(9.2 \%)$ were ALDH1 positive. Similar to Set $1, \mathrm{CD} 44+/ \mathrm{CD} 24-$ was positively correlated with ALDH1 expression $(r h o=0.137, P=0.004)$. In Set $2, \mathrm{CD} 44+/ \mathrm{CD} 24-$ was associated with hormone receptor negativity $(P=0.001)$, basal-like phenotype $(P<0.001)$, and absence of nodal metastasis $(P=0.024)$. The ALDH1 positivity tended to be associated with hormone receptor negativity $(P=0.067)$, basal-like phenotype $(P=0.050)$, and lymphovascular invasion $(P=0.063$; Supplementary Table S2).

At the time of analysis, the median follow-up period was 40.7 (5.6-104.0) months, during which there were 34 (7.6\%) recurrences and 25 (5.6\%) deaths. In comparison with the patients treated with adjuvant chemotherapy alone in Set 1 , the patients in this group showed significantly favourable disease-free and overall survivals (5-year disease-free survival rate, $81.4 \%$ vs $91.2 \%$, $P=0.001$; overall 5-year survival rate, $85.8 \%$ vs $93.1 \%$, $P=0.017$ ). In univariate survival analyses (Table 3 ), patients with CD44 + /CD24 - phenotype had a shorter disease-free survival time than patients without expression of this marker $(P=0.005$; Figure 3); however, CD44+/CD24 - expression was not associated with overall survival of the patients $(P=0.181$;
Figure 3). The ALDH1 positivity had no impact on the clinical outcome of the patients (disease-free survival, $P=0.748$; overall survival, $P=0.322$; Figure 3 ).

Beside CD44+/CD24 - expression, pT stage, pN stage, lymphovascular invasion, and basal-like phenotype were associated with clinical outcome of the patients in univariate analyses (Table 3). In multivariate analysis adjusted for $\mathrm{pT}$ stage, pN stage, lymphovascular invasion, hormone receptor status, and basal-like phenotype, CD44+/CD24 - expression $(\mathrm{HR}=2.213$, $95 \% \mathrm{CI}=1.085-4.515, P=0.029)$ and lymphovascular invasion $(\mathrm{HR}=2.694, \quad 95 \% \quad \mathrm{CI}=1.304-5.566, \quad P=0.007)$ remained as independent negative prognostic factors for disease-free survival (Table 3).

In subgroup analysis of Set 2 by hormone receptor status, a prognostic effect of CD $44+/ C D 24-$ phenotype was found in the hormone receptor-negative cases $(P=0.021)$, but not in hormone receptor-positive cases $(P=0.274)$. Multivariate analyses including CD44 + /CD24 - phenotype, pT stage, pN stage, lymphovascular invasion, and basal-like phenotype revealed that CD $44+/ \mathrm{CD} 24-$ phenotype $(\mathrm{HR}=2.679,95 \% \mathrm{CI}=1.101-6.520, P=0.030)$ and pT stage $(\mathrm{HR}=2.872,95 \% \mathrm{CI}=1.132-7.285, P=0.026)$ were independent prognostic factors for disease-free survival in hormone receptor-negative subgroup (Table 3 ).

Relationship between BCSC marker expression and response to trastuzumab-based therapy in HER2-positive metastatic breast cancer (Set 3). We also explored the prognostic and predictive value of CD44 +/CD24 - and ALDH1 expression in 112 cases of HER2-positive metastatic breast cancer treated with trastuzumabbased chemotherapy. Of the 102 cases (tissue samples were inadequate in 10 cases including the 3 cases overlapped with Set 1 ), expression of CD44 +/CD24 - and ALDH1 was found in $29.4 \%$ (30/102) and $9.8 \%(10 / 102)$, respectively. Their expression was positively correlated $(r h o=0.221, P=0.025)$. The $\mathrm{CD} 44+1$ CD24 - expression also showed an association with hormone receptor negativity $(P=0.005)$ and basal-like phenotype $(P=0.001)$; ALDH1 positivity was associated with hormone receptor negativity $(P=0.046)$, and tended to be associated with basal-like phenotype $(P=0.085$; Supplementary Table S3). However, expression of CD44+/CD24 - and ALDH1 was not associated with clinical benefit of trastuzumab treatment ( $P=0.759$ and $P=0.142$, respectively). In patient outcomes, $\mathrm{CD} 44+/ \mathrm{CD} 24-$ expression was not associated with progression-free or overall survival $(P=0.356$ and $P=0.738$, respectively; Supplementary Figure S1); findings for ALDH1 expression were similar $(P=0.871$ and $P=0.447$, respectively; Supplementary Figure $\mathrm{S} 1)$.

Table 3. Univariate and multivariate analyses of disease-free survival in HER2-positive primary breast cancer patients treated with adjuvant trastuzumab in Set 2

\begin{tabular}{|c|c|c|c|c|c|c|c|}
\hline \multirow[b]{2}{*}{ Variable } & \multirow[b]{2}{*}{ Category } & \multicolumn{3}{|c|}{ Univariate analysis } & \multicolumn{3}{|c|}{ Multivariate analysis } \\
\hline & & HR & $95 \% \mathrm{Cl}$ & $P$-value & HR & $95 \% \mathrm{Cl}$ & $P$-value \\
\hline \multicolumn{8}{|l|}{ Whole population } \\
\hline pN stage & No vs N1-3 & 2.488 & $1.026-6.032$ & 0.037 & 1.942 & $0.773-4.878$ & 0.158 \\
\hline Lymphovascular invasion & Absent vs present & 2.985 & $1.455-6.125$ & 0.002 & 2.694 & $1.304-5.566$ & 0.007 \\
\hline Hormone receptor status & Negative vs positive & 0.547 & $0.267-1.123$ & 0.095 & 0.717 & $0.321-1.602$ & 0.417 \\
\hline Basal-like phenotype & Negative vs positive & 2.238 & $1.142-4.385$ & 0.016 & 2.002 & $1.001-4.002$ & 0.050 \\
\hline \multicolumn{8}{|c|}{ Hormone receptor-negative subgroup } \\
\hline pT stage & T1 vs T2-4 & 2.983 & $1.176-7.568$ & 0.016 & 2.872 & $1.132-7.285$ & 0.026 \\
\hline $\mathrm{pN}$ stage & No vs N1-3 & 2.002 & $0.741-5.408$ & 0.163 & 1.606 & $0.569-4.534$ & 0.371 \\
\hline Lymphovascular invasion & Absent vs present & 2.378 & $1.029-5.495$ & 0.037 & 1.982 & $0.849-4.625$ & 0.114 \\
\hline Basal-like phenotype & Negative vs positive & 1.760 & $0.762-4.068$ & 0.180 & 1.461 & $0.619-3.449$ & 0.387 \\
\hline CD44 +/CD24 - expression & Negative vs positive & 2.728 & $1.121-6.638$ & 0.021 & 2.679 & $1.101-6.520$ & 0.030 \\
\hline
\end{tabular}


A

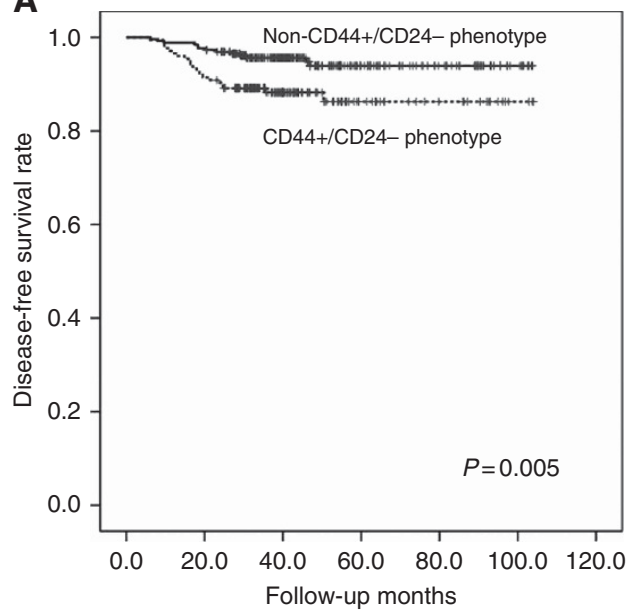

C

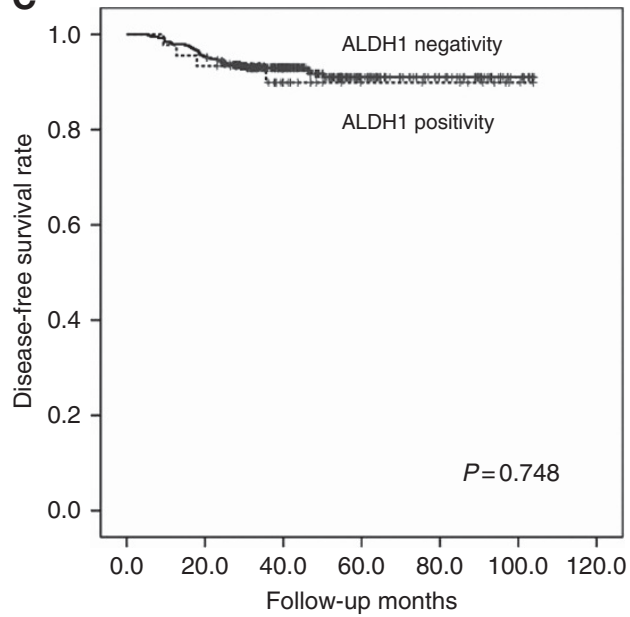

B

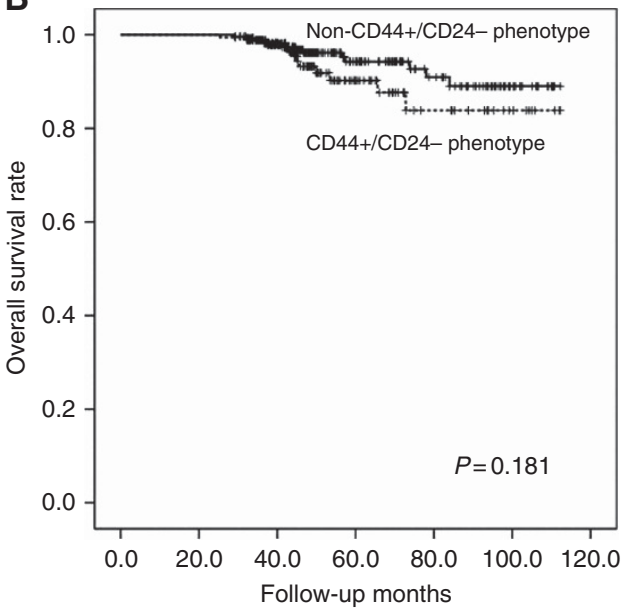

D

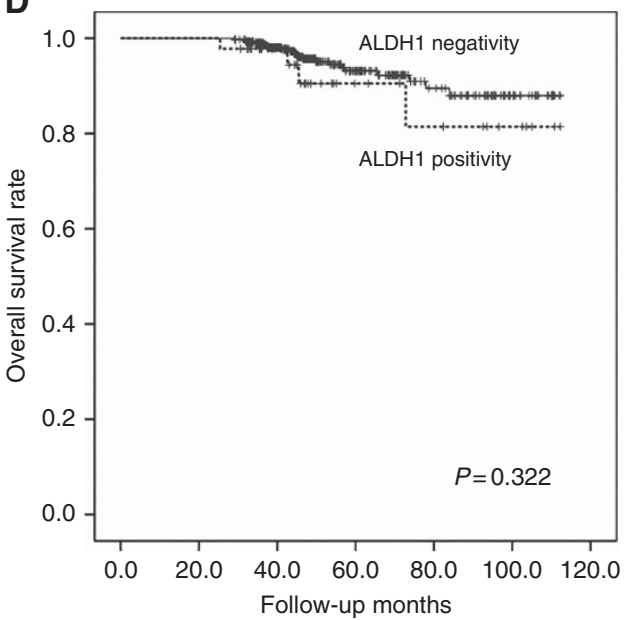

Figure 3. Kaplan-Meier plots of survival according to expression of CD44 + /CD24 - and ALDH1 in HER2-positive primary breast cancer patients treated with adjuvant trastuzumab (Set 2). The CD44 +/CD24 - phenotype is associated with poor disease-free survival (A) but not with overall survival (B). The ALDH1 positivity shows no impact on disease-free (C) or overall survival (D).

\section{DISCUSSION}

Primary (de novo) resistance to trastuzumab is not infrequent in clinically HER2-positive breast cancers. There are increasing demands, therefore, to identify the molecular mechanisms underlying trastuzumab resistance and to develop successful therapeutic targets that overcome the resistance in HER2-positive breast cancer. Here, we have shown that expression of BCSC markers, particularly $\mathrm{CD} 44+/ \mathrm{CD} 24-$ phenotype, is an independent factor for poor prognosis in patients with HER2-positive primary breast cancers receiving adjuvant trastuzumab, suggesting that BCSC marker expression has a predictive value for trastuzumab response. We also revealed that $\mathrm{CD} 44+/ \mathrm{CD} 24-$ phenotype is an independent factor for poor prognosis in patients with HER2-positive primary breast cancer not treated with adjuvant trastuzumab, indicating that the BCSC phenotype is associated with poor clinical outcome in HER2-positive breast cancer. To the best of our knowledge, there have been no large studies investigating the prognostic and predictive significance of BCSC marker expression in HER2-positive breast cancer samples. Our findings imply an important role for BCSCs, affecting patient outcome and response to HER2-targeted therapy in HER2-positive primary breast cancers.

The intertumoral phenotypic and molecular heterogeneity of breast cancer presents a considerable challenge for development of effective strategies for preventing and treating this disease (Kakarala and Wicha, 2008). In particular, BCSCs have been thought to be an obstacle to successful therapy of breast cancer because of their highly tumourigenic and metastatic potential, and their chemoresistance (Clarke and Fuller, 2006; Polyak and Hahn, 2006; Kakarala and Wicha, 2008; Liu et al, 2014). It has been suggested that success in HER2-targeted therapies may be driven by direct activity against HER2-positive BCSCs (Bedard et al, 2009; Martin-Castillo et al, 2013). In that respect, Korkaya et al (2008) demonstrated that trastuzumab efficacy was directly associated with its effect on the BCSC subpopulation by showing that the drug decreased the ALDEFLUOR-positive population in trastuzumab-sensitive HER2-positive breast cancer cell lines. Moreover, Li et al (2008) reported that lapatinib, a dual EGFR/HER2 inhibitor, led to a decrease in the percentage of CD44+/CD24 - cells and in efficiency of mammosphere formation after treatment, suggesting that specific pathway inhibitors may provide a therapeutic strategy for eliminating BCSCs.

However, although it has been suggested that the success of HER2-targeted therapy, such as with trastuzumab, is associated with its effect on BCSCs, failure of HER2-targeted therapy may also be driven by BCSCs. That is, the variety of possible mechanisms of escape from trastuzumab involve many of the same markers that have been implicated in the biology of BCSCs (Bedard et al, 2009). For example, overexpression of BCSC marker CD44 may lead to loss or blockade of the trastuzumab-binding site 
at the HER2 extracellular domain (Martin-Castillo et al, 2013). Furthermore, the increased expression of CXCR 4 and $\beta 1$ integrin in BCSCs can lead to activation of alternative pathways circumventing HER2 signalling (Martin-Castillo et al, 2013). In HER2-positive cell lines, spontaneous epithelial-mesenchymal transition, which has been linked to the acquisition of BCSC properties (Mani et al, 2008), was associated with CD $44+1$ CD24 - expression and progressive loss of membrane HER2 expression (Oliveras-Ferraros et al, 2010; Lesniak et al, 2013). The involvement of BCSCs in trastuzumab resistance is supported by our finding that CD44+/CD24 - expression was associated with poor clinical outcome in patients with HER2-positive primary breast cancer treated with adjuvant trastuzumab. However, we also showed that CD $44+/ \mathrm{CD} 24-$ expression was a poor prognostic factor in HER2-positive primary breast cancer patients not treated with adjuvant trastuzumab. Thus, the association of $\mathrm{CD} 44+1$ CD24 - with poor clinical outcome in patients receiving adjuvant trastuzumab cannot be explained only by BCSC-driven resistance to the drug, but is also explained by the prognostic significance of the BCSC phenotype itself. Establishment of the role of BCSCs in trastuzumab resistance in HER2-positive breast cancer requires further comprehensive studies in neoadjuvant settings.

In the present study, expression of CD44+/CD24- and ALDH1 was not associated with response to trastuzumab or survival in patients with HER2-positive metastatic breast cancer. There are some limitations in this metastatic set, as mentioned in a previous report (Lee et al, 2014b). Not all the patients with metastatic cancer were treated according to the same protocol, and the tissues used for analyses were not homogeneous, as some were from primary sites and others from metastatic sites. Extensive studies are therefore necessary to identify whether expression of BCSC markers predicts response to trastuzumab in HER2-positive metastatic breast cancer.

According to recent meta-analyses, expression of CD $44+1$ CD24 - and ALDH1 shows a close correlation with hormone receptor negativity (Zhou et al, 2010; Liu et al, 2014). Similar results were found in the present study, where CD44+/CD24and ALDH1 expression was associated with hormone receptor negativity in all three sets of cases. It is now generally accepted that clinically HER2-positive breast cancers are not homogeneous, as there are at least two subgroups according to their hormone receptor status. Regarding clinical behaviour, in the era before HER2-targeted therapy, patients with oestrogen receptor (ER)-negative/HER2-positive breast cancer had worse prognosis than those with ER-positive/HER2-positive breast cancer (Chia et al, 2008). We showed in a previous study that the histopathologic features of HER2-positive breast cancer differ according to hormone receptor status, with high histologic grade, heavy lymphocytic infiltration, high level of HER2 gene amplification, and p53 overexpression in hormone receptor-negative/HER2-positive breast cancers (Lee et al, 2014a).

In addition to hormone receptor status, basal phenotype appears to define a new subgroup of HER2-positive breast cancers. Bagaria et al (2012) classified HER2-positive breast cancer into three subgroups based on ER status and basal CK expression (luminal-HER2 + (ER-positive/basal CK-negative), HER2 + (ERnegative/basal CK-negative), and basal-HER2 + (ER-negative/ basal CK-positive)) and found that the basal-HER2 + type was independently associated with poor survival (Bagaria et al, 2012). Moreover, it has been suggested that basal-like HER2-positive breast cancers are more likely to be intrinsically resistant to trastuzumab (Harris et al, 2007; Oliveras-Ferraros et al, 2010; Martin-Castillo et al, 2013). The present study supports the previous studies by showing that basal-like phenotype was associated with poor clinical outcome in patients with HER2positive primary breast cancer treated with adjuvant trastuzumab. The BCSC phenotype, especially CD $44+/ \mathrm{CD} 24-$, is closely related to basal-like breast cancer (Honeth et al, 2008; Park et al, 2010). Moreover, in this study, BCSC marker expression was also closely correlated with basal-like phenotype in HER2-positive breast cancer. Thus, the association of CD 44+/CD24expression with trastuzumab resistance and poor clinical outcome may be explained by its close relationship with the basal-like phenotype. In the light of these findings, as mentioned above, HER2-positive breast cancers need to be redefined within a new molecular taxonomy.

In summary, our results provided evidence for the prognostic and predictive value of BCSCs in HER2-positive primary breast cancer. Expression of $\mathrm{CD} 44+/ \mathrm{CD} 24$ was found to be an independent negative indicator for prognosis and a predictive marker for trastuzumab response in HER2-postive primary breast cancers, although not in metastatic breast cancer. Further investigations on the clinical implications of BCSCs in HER2positive breast cancer, together with effective targeting of BCSCs, might contribute to improving the clinical outcome of patients.

\section{ACKNOWLEDGEMENTS}

This research was supported by the Basic Science Research Program through the National Research Foundation of Korea (NRF) funded by the Ministry of Science, ICT and Future Planning (Grant No. NRF-2012R1A1B4000557).

\section{CONFLICT OF INTEREST}

The authors declare no conflict of interest.

\section{REFERENCES}

Al-Hajj M, Wicha MS, Benito-Hernandez A, Morrison SJ, Clarke MF (2003) Prospective identification of tumorigenic breast cancer cells. Proc Natl Acad Sci USA 100: 3983-3988.

Bagaria SP, Ray PS, Wang J, Kropcho L, Chung A, Sim MS, Shamonki JM, Martino S, Cui X, Giuliano AE (2012) Prognostic value of basal phenotype in HER2-overexpressing breast cancer. Ann Surg Oncol 19: 935-940.

Bedard PL, Cardoso F, Piccart-Gebhart MJ (2009) Stemming resistance to HER-2 targeted therapy. J Mammary Gland Biol Neoplasia 14: 55-66.

Chia S, Norris B, Speers C, Cheang M, Gilks B, Gown AM, Huntsman D, Olivotto IA, Nielsen TO, Gelmon K (2008) Human epidermal growth factor receptor 2 overexpression as a prognostic factor in a large tissue microarray series of node-negative breast cancers. J Clin Oncol 26: 5697-5704.

Clarke MF, Fuller M (2006) Stem cells and cancer: two faces of eve. Cell 124: 1111-1115.

Eisenhauer EA, Therasse P, Bogaerts J, Schwartz LH, Sargent D, Ford R, Dancey J, Arbuck S, Gwyther S, Mooney M, Rubinstein L, Shankar L, Dodd L, Kaplan R, Lacombe D, Verweij J (2009) New response evaluation criteria in solid tumours: revised RECIST guideline (version 1.1). Eur J Cancer 45: 228-247.

Ginestier C, Hur MH, Charafe-Jauffret E, Monville F, Dutcher J, Brown M, Jacquemier J, Viens P, Kleer CG, Liu S, Schott A, Hayes D, Birnbaum D, Wicha MS, Dontu G (2007) ALDH1 is a marker of normal and malignant human mammary stem cells and a predictor of poor clinical outcome. Cell Stem Cell 1: 555-567.

Harris LN, You F, Schnitt SJ, Witkiewicz A, Lu X, Sgroi D, Ryan PD, Come SE, Burstein HJ, Lesnikoski BA, Kamma M, Friedman PN, Gelman R, Iglehart JD, Winer EP (2007) Predictors of resistance to preoperative trastuzumab and vinorelbine for HER2-positive early breast cancer. Clin Cancer Res 13: 1198-1207.

Honeth G, Bendahl PO, Ringner M, Saal LH, Gruvberger-Saal SK, Lovgren K, Grabau D, Ferno M, Borg A, Hegardt C (2008) The CD44 + /CD24 phenotype is enriched in basal-like breast tumors. Breast Cancer Res 10: R53. 
Kakarala M, Wicha MS (2008) Implications of the cancer stem-cell hypothesis for breast cancer prevention and therapy. J Clin Oncol 26: 2813-2820.

Korkaya H, Paulson A, Iovino F, Wicha MS (2008) HER2 regulates the mammary stem/progenitor cell population driving tumorigenesis and invasion. Oncogene 27: 6120-6130.

Lee HE, Kim JH, Kim YJ, Choi SY, Kim SW, Kang E, Chung IY, Kim IA, Kim EJ, Choi Y, Ryu HS, Park SY (2011) An increase in cancer stem cell population after primary systemic therapy is a poor prognostic factor in breast cancer. Br J Cancer 104: 1730-1738.

Lee HJ, Park IA, Park SY, Seo AN, Lim B, Chai Y, Song IH, Kim NE, Kim JY, Yu JH, Ahn JH, Gong G (2014a) Two histopathologically different diseases: hormone receptor-positive and hormone receptor-negative tumors in HER2-positive breast cancer. Breast Cancer Res Treat 145: 615-623.

Lee HJ, Seo AN, Kim EJ, Jang MH, Kim YJ, Kim JH, Kim SW, Ryu HS, Park IA, Im SA, Gong G, Jung KH, Kim HJ, Park SY (2014b) Prognostic and predictive values of EGFR overexpression and EGFR copy number alteration in HER2-positive breast cancer. Br J Cancer 112(1): 103-111.

Lee HJ, Seo AN, Kim EJ, Jang MH, Suh KJ, Ryu HS, Kim YJ, Kim JH, Im SA, Gong G, Jung KH, Park IA, Park SY (2014c) HER2 heterogeneity affects trastuzumab responses and survival in patients with HER2-positive metastatic breast cancer. Am J Clin Pathol 142: 755-766.

Lesniak D, Sabri S, Xu Y, Graham K, Bhatnagar P, Suresh M, Abdulkarim B (2013) Spontaneous epithelial-mesenchymal transition and resistance to HER-2-targeted therapies in HER-2-positive luminal breast cancer. PLoS One 8: e71987.

Li X, Lewis MT, Huang J, Gutierrez C, Osborne CK, Wu MF, Hilsenbeck SG, Pavlick A, Zhang X, Chamness GC, Wong H, Rosen J, Chang JC (2008) Intrinsic resistance of tumorigenic breast cancer cells to chemotherapy. J Natl Cancer Inst 100: 672-679.

Liu Y, Lv DL, Duan JJ, Xu SL, Zhang JF, Yang XJ, Zhang X, Cui YH, Bian XW, Yu SC (2014) ALDH1A1 expression correlates with clinicopathologic features and poor prognosis of breast cancer patients: a systematic review and meta-analysis. BMC Cancer 14: 444.

Mani SA, Guo W, Liao MJ, Eaton EN, Ayyanan A, Zhou AY, Brooks M, Reinhard F, Zhang CC, Shipitsin M, Campbell LL, Polyak K, Brisken C, Yang J, Weinberg RA (2008) The epithelial-mesenchymal transition generates cells with properties of stem cells. Cell 133: 704-715.

Martin-Castillo B, Oliveras-Ferraros C, Vazquez-Martin A, Cufi S, Moreno JM, Corominas-Faja B, Urruticoechea A, Martin AG, Lopez-Bonet E, Menendez JA (2013) Basal/HER2 breast carcinomas: integrating molecular taxonomy with cancer stem cell dynamics to predict primary resistance to trastuzumab (Herceptin). Cell Cycle 12: 225-245.

Mukohara T (2011) Mechanisms of resistance to anti-human epidermal growth factor receptor 2 agents in breast cancer. Cancer Sci 102: 1-8.

Nahta R, Yu D, Hung MC, Hortobagyi GN, Esteva FJ (2006) Mechanisms of disease: understanding resistance to HER2-targeted therapy in human breast cancer. Nat Clin Pract Oncol 3: 269-280.

Nalwoga H, Arnes JB, Wabinga H, Akslen LA (2010) Expression of aldehyde dehydrogenase 1 (ALDH1) is associated with basal-like markers and features of aggressive tumours in African breast cancer. $\mathrm{Br} J$ Cancer 102: 369-375.

Oliveras-Ferraros C, Vazquez-Martin A, Martin-Castillo B, Cufi S, Del Barco S, Lopez-Bonet E, Brunet J, Menendez JA (2010) Dynamic emergence of the mesenchymal CD44(pos)CD24(neg/low) phenotype in HER2-gene amplified breast cancer cells with de novo resistance to trastuzumab (Herceptin). Biochem Biophys Res Commun 397: 27-33.

Park SY, Lee HE, Li H, Shipitsin M, Gelman R, Polyak K (2010) Heterogeneity for stem cell-related markers according to tumor subtype and histologic stage in breast cancer. Clin Cancer Res 16: 876-887.

Polyak K, Hahn WC (2006) Roots and stems: stem cells in cancer. Nat Med 12: 296-300.

Press MF, Pike MC, Chazin VR, Hung G, Udove JA, Markowicz M, Danyluk J, Godolphin W, Sliwkowski M, Akita R, Paterson M, Slamon DJ (1993) Her-2/neu expression in node-negative breast cancer: direct tissue quantitation by computerized image analysis and association of overexpression with increased risk of recurrent disease. Cancer Res 53: 4960-4970.

Romond EH, Perez EA, Bryant J, Suman VJ, Geyer Jr CE, Davidson NE, Tan-Chiu E, Martino S, Paik S, Kaufman PA, Swain SM, Pisansky TM, Fehrenbacher L, Kutteh LA, Vogel VG, Visscher DW, Yothers G, Jenkins RB, Brown AM, Dakhil SR, Mamounas EP, Lingle WL, Klein PM, Ingle JN, Wolmark N (2005) Trastuzumab plus adjuvant chemotherapy for operable HER2-positive breast cancer. $N$ Engl J Med 353: $1673-1684$.

Slamon DJ, Clark GM, Wong SG, Levin WJ, Ullrich A, McGuire WL (1987) Human breast cancer: correlation of relapse and survival with amplification of the HER-2/neu oncogene. Science 235: 177-182.

Slamon DJ, Leyland-Jones B, Shak S, Fuchs H, Paton V, Bajamonde A, Fleming T, Eiermann W, Wolter J, Pegram M, Baselga J, Norton L (2001) Use of chemotherapy plus a monoclonal antibody against HER2 for metastatic breast cancer that overexpresses HER2. N Engl J Med 344: 783-792.

Tandon AK, Clark GM, Chamness GC, Ullrich A, McGuire WL (1989) HER-2/neu oncogene protein and prognosis in breast cancer. J Clin Oncol 7: $1120-1128$

Tanei T, Morimoto K, Shimazu K, Kim SJ, Tanji Y, Taguchi T, Tamaki Y, Noguchi S (2009) Association of breast cancer stem cells identified by aldehyde dehydrogenase 1 expression with resistance to sequential Paclitaxel and epirubicin-based chemotherapy for breast cancers. Clin Cancer Res 15: 4234-4241.

Zhou L, Jiang Y, Yan T, Di G, Shen Z, Shao Z, Lu J (2010) The prognostic role of cancer stem cells in breast cancer: a meta-analysis of published literatures. Breast Cancer Res Treat 122: 795-801.

This work is published under the standard license to publish agreement. After 12 months the work will become freely available and the license terms will switch to a Creative Commons AttributionNonCommercial-Share Alike 4.0 Unported License.

Supplementary Information accompanies this paper on British Journal of Cancer website (http://www.nature.com/bjc) 